\title{
Phytochemicals and potential therapeutic targets on toxoplasma gondii parasite
}

\begin{abstract}
Identification of drug target in protozoan T. gondii is an important step in the development of chemotherapeutic agents. Likewise, exploring phytochemical compounds effective against the parasite can lead to the development of new drug agent that can be useful for prophylaxis and treatment of toxoplasmosis. In this review, we searched for the relevant literature on the herbs that were tested against T. gondii either in vitro or in vivo, as well as different phytochemicals and their potential activities on T. gondii. Potential activities of major phytochemicals, such as alkaloid, flavonoid, terpenoids and tannins on various target sites on $\mathrm{T}$. gondii as well as other related parasites was discussed. It is believed that the phytochemicals from natural sources are potential drug candidates for the treatment of toxoplasmosis with little or no toxicity to humans.
\end{abstract}

Keyword: Phytochemicals; Drug target; Parasite; T. gondii; Toxoplasmosis; In vivo; In vitro 